\title{
The Orphan Receptor GPR88 Controls Impulsivity and Is a Risk Factor for Attention- Deficit/Hyperactivity Disorder
}

Brigitte Kieffer ( $\nabla$ brigitte.kieffer@unistra.fr )

McGill University https://orcid.org/0000-0002-8809-8334

Sami Ben Hamida

Douglas Mental Health University Institute https://orcid.org/0000-0001-9636-9432

Sarojini Sengupta

McGill University

Emmie Clarke

McGill University

Michael McNicholas

McGill University

Eleonara Moroncini

McGill University

Emmanuel Darcq

McGill University https://orcid.org/0000-0002-8377-8015

Marina Ter-Stepanian

McGill University

Marie-Eve Fortier

McGill University

Natalie Grizenko

McGill University

Ridha Joober

McGill University and Douglas Hospital Research Centre

Article

Keywords:

Posted Date: January 25th, 2022

DOI: https://doi.org/10.21203/rs.3.rs-1260604/v1 
License: (c) (i) This work is licensed under a Creative Commons Attribution 4.0 International License. Read Full License

Version of Record: A version of this preprint was published at Molecular Psychiatry on September 8th, 2022. See the published version at https://doi.org/10.1038/s41380-022-01738-w. 


\section{Abstract}

The neural orphan G protein coupled receptor GPR88 is predominant in the striatum and cortex of both rodents and humans, and considered a potential target for brain disorders. Previous studies have shown multiple behavioral phenotypes in Gpr88 knockout mice, and human genetic studies have reported association with psychosis. Here we tested the possibility that GPR88 contributes to Attention Deficit Hyperactivity Disorder (ADHD). In the mouse, we tested Gpr88 knockout mice in three behavioral paradigms, best translatable between rodents and humans, and found higher motor impulsivity and reduced attention together with the reported hyperactivity. Atomoxetine, a typical ADHD drug, reduced impulsivity in mutant mice. Conditional Gpr88 knockout mice in either D1R-type or D2R-type medium spiny neurons revealed distinct implications of the two receptor populations in waiting and stopping impulsivity. Thus, animal data demonstrate that deficient GPR88 activity causally promotes ADHD-like behaviors, and identify circuit mechanisms underlying GPR88-regulated impulsivity. In humans, we performed a family-based genetic study including 567 nuclear families with DSM-IV diagnosis of ADHD. There was a minor association for SNP rs2036212 with diagnosis, treatment response and cognition. A stronger association was found for SNP rs2809817 upon patient stratification, suggesting that the T allele is a risk factor when prenatal stress is involved. Human data therefore identify GPR88 variants associated with the disease, and highlight a potential role of life trajectories to modulate GPR88 function. Overall, animal and human data concur to suggest that GPR88 signaling should be considered a key factor for diagnostic and treatment of ADHD.

\section{Brief Summary}

Mutant mouse behavior and human genetic data concur to suggest that the orphan receptor GPR88 contributes to Attention-Deficit/ Hyperactivity Disorder.

\section{Introduction}

GPR88 is a brain orphan G protein coupled receptor (GPCR), with predominant striatal and cortical expression in both rodents and humans ${ }^{1,2}$. In preclinical research, current knowledge of GPR88 function is mostly based on behavioral analyses of genetically modified mice lacking the Gpr88 gene constitutively (or Gpr88 knockout mice). These mutant mice show a complex endophenotype, and most prominent deficits include motor hyperactivity and motor coordination deficits ${ }^{3-5}$, failure to habituate and risk-taking behavior ${ }^{5-7}$, impaired sensorimotor gating ${ }^{3}$ and sensory processing ${ }^{1}$, reduced working memory and cognitive flexibility ${ }^{7}$, as well as increased alcohol seeking ${ }^{8}$. Resting-state functional Magnetic Resonance Imaging of these mutant mice shows broad remodeling of brain connectivity ${ }^{9}$. In brief, major modifications were identified for the retrosplenial cortex, central to the Default Mode Network, as well as somatosensory and motor cortical networks, likely related to their hyperactivity and sensory deficits. Also weakened connectivity of sensorymotor cortex with amygdala, and prefrontal cortex with mesolimbic circuits paralleled risk-taking ${ }^{5}$ and high alcohol drinking ${ }^{8}$ behaviors, respectively. Finally, 
stronger correlation of motor cortex and caudate putamen activities was consistent with impaired motor coordination and altered habit learning ${ }^{5}$ in these animals. Altogether, both behavioral studies and neuroimaging concur to demonstrate multiple GPR88 functions in the brain, hence the potential of GPR88 as a target for neuropsychiatric disorders is promising. Pharmacology is still at an early stage, and a first GPR88 agonist showed in vivo efficacy in reducing alcohol drinking ${ }^{10}$, consistent with the high drinking phenotype of Gpr88 knockout mice.

Cellular mechanisms underlying GPR88 function have focused on the striatum. In this brain structure, GPR88 is almost equally distributed between medium spiny neurons (MSNs) of the direct (expressing dopamine D1 receptors or D1R-MSNs) and indirect (expressing dopamine D2 receptors or D2R-MSNs) pathways ${ }^{4}$, known to exert opposing influences on motor output systems. We previously showed that conditional deletion of the Gpr88 gene in either D1R- or D2R-MSNs demonstrates dissociable roles of GPR88 in the two pathways ${ }^{11}$. In brief, D2R-MSN GPR88 mutant mice recapitulated most phenotypes of constitutive knockout animals, including high levels of locomotion and stereotypies, impaired initial motor coordination, defensive burying and social approach, while the D1R-MSN mutants specifically showed lack of habituation and motor skill learning deficits. The cell-type specificity of GPR88 function also extends to sensory gating ${ }^{12}$ and anxiety-like behaviors ${ }^{13}$. Thus, distinct cellular and circuit mechanisms subserve the complex GPR88 function in the brain.

Studies in humans remain limited. A study identified a deleterious homozygous mutation in the GPR88 gene in a consanguineous family, affecting four sisters with childhood chorea, learning disabilities and speech retardation ${ }^{14}$. Another genetic study performed in three different populations found a positive association between single nucleotide polymorphisms (SNPs) of the GPR88 gene and bipolar disorder in Sardinian and Palestinian triads, and with schizophrenia in triads from the Xhosa population in South Africa $^{15,16}$. Also, a genome wide bioinformatics analysis of miRNA-mRNA pairs identified a 3'UTR GPR88 variant possibly associated with intellectual disability ${ }^{17}$. These studies therefore reveal the relevance of GPR88 as a risk factor for brain development and psychosis, supporting the search for association with perhaps other neuropsychiatric disorders.

In this report, we examine the possibility that GPR88 is implicated in the etiology of AttentionDeficit/Hyperactivity Disorder (ADHD), a highly prevalent developmental psychiatric condition ${ }^{18}$. ADHD affects children and, when left untreated, is associated to adverse consequences in adulthood, including drug abuse, anxiety, depressed mood, disturbance of emotions, and social rejection ${ }^{19-21}$. The disease is characterized by three main symptoms, namely hyperactivity, inattention and impulsivity (Fig. 1). In animal research, several behavioral models with face and/or predictive validity have been proposed to address these ADHD-like core symptoms ${ }^{22}$. Here, we first tested Gpr88 knockout mice in three behavioral paradigms considered best translatable between rodents and humans for ADHD-like symptoms (Fig. 1), and found deficits in all the tests. Second, we found that a typical ADHD drug used in the clinic, atomoxetine, reduces the ADHD-like phenotype of mutant mice. Third, we tested conditional D1R-MSN and D2R-MSN Gpr88 knockout mice and discovered pathway-specific mechanisms of GPR88 function in 
the regulation of motor impulsivity. Fourth, we performed a family-based genetic study in a clinical sample of children with ADHD, covering six GPR88SNPs, and found a significant association with multiple dimensions of ADHD, particularly under conditions of prenatal stress.

\section{Methods}

\section{The animal study}

Mice. Total Gpr88 ${ }^{-/-}$mice were produced as described in Ref ${ }^{5}$. Conditional A2AR-Gpr88 and D1R-Gpr88 mice lacking GPR88 in D2R- and D1R-MSNs, respectively, were produced as described in Ref ${ }^{11}$. The mice were bred at the Douglas Research Centre. All animals were group-housed with littermates (2-5 animals per cage) under a $12 \mathrm{~h}$ light/dark cycle and given food and water ad libitum unless otherwise stated.

Behavior. Procedures used for the 5-Choice Reaction Time Task (5-CSRTT), Attentional Set Shifting Task (ASST), Go-NoGo task, atomoxetine administration and statistical analysis of the animal study are detailed in the Suppl Methods section. Statistical analysis of the data is detailed in Suppl Tables S1 and S2.

\section{The human study}

Participants and evaluations: ADHD. Children (between 6 and 12 years of age, mean=9 years) with a diagnosis of ADHD, were recruited from the Disruptive Behavior Disorders Program and the child psychiatry outpatient clinics at the Douglas Mental Health University Institute (DMHUI) in Montreal. They were referred to these specialized care facilities by schools, community social workers, family doctors and pediatricians. The research protocol was approved by the DMHUI Research Ethics Board. Parents were explained the study and provided written consent. Children (affected child and unaffected siblings) were explained the study and gave their assent to participate. Children with an IQ less than 70, and/or a diagnosis of either Tourette syndrome, pervasive developmental disorder, or psychosis were excluded from the study.

Details of the pharmaco-behavioural-genetic study and the different assessments conducted have been previously described ${ }^{23}$. Briefly, the diagnosis (based on DSM-IV criteria) was based on clinical interviews between the child psychiatrist, the affected child and at least one parent. This clinical examination was supplemented with a structured clinical interview of parents using the Diagnostic Interview Schedule for Children-version IV, DISC-IV24 and school reports including the Conners' Global Index-Teacher version questionnaire (Conners'-T) ${ }^{25}$. The child's behaviour at home was evaluated using the Conners' Global Index-Parents (Conners'-P) ${ }^{26}$ and the Child Behaviour Checklist $(\mathrm{CBCL})^{27}$. In the majority of cases, mothers were the primary informants. These evaluations were completed while the child was not taking any medication.

Cognitive dimensions, specifically those related to executive function (EF), were included as quantitative traits in the genetic association analyses. EF encapsulates the range of cognitive abilities that are 
required for completing a given task, and include response inhibition, sustained attention, working memory, set-shifting, planning and organization. The neuropsychological tests conducted were: Conners' Continuous Performance Test (CPT: measures attention, response inhibition, and impulse control) ${ }^{28}$, SelfOrdered Pointing Task (SOPT: visual working memory, planning, and response inhibition) ${ }^{29}$, Wisconsin Card Sorting Test (WCST: measure of cognitive flexibility and set-shifting) (Heaton, Chelune, Talley, Kay, \& Curtiss, 1993), and Tower of London test (TOL: planning, organization, and problem-solving capacity) ${ }^{30}$. These tests were performed as described ${ }^{23,31}$. These neuropsychological assessments were carried out at the end of a one-week washout period if the child was on medication prior to inclusion in the study. In addition to the measures of EF, IQ (full scale, verbal, and performance IQ) was evaluated using the Wechsler Intelligence Scale (WISC-III/IV) ${ }^{32}$.

Obstetrical (pregnancy, delivery and perinatal) complications were assessed using the Kinney Medical and Gynecological Questionnaire and scored using the McNeil-Sjöström scale ${ }^{33}$. Mothers were also asked to describe stressful life events experienced during the pregnancy, if any. This information was used to score maternal stress levels from 1 to 4 based on the DSM-III and DSM-III-R axis IV scales (1 = no stress, 2 = mild, 3 = moderate, 4 = severe). Examples of mild stress include events such as arguments with friends; moderate/ severe stressors include separation from partner, repeated physical or sexual abuse, imprisonment of a spouse, or death of a very close relative. Since information on environmental factors was collected retrospectively (and may therefore be subject to recall bias), the information provided by the mother was corroborated in an independent interview with a second individual close to the mother wherever possible.

Response to treatment with MPH was assessed in a double-blind, placebo-controlled, within-subject (crossover) randomized control trial conducted over a two-week period, as described ${ }^{23}$ (trial registration number: NCT00483106). Briefly, subjects received one week of treatment with placebo (PBO) and one week of treatment with $0.5 \mathrm{mg} / \mathrm{kg}$ of $\mathrm{MPH}$ in a divided b.i.d. dose $(0.25 \mathrm{mg} / \mathrm{kg}$, morning and noon), following a wash-out period. At the end of each week of treatment, the parents and teacher were asked to evaluate the behaviour of the child using the Conners'-parents and Conners'-teachers respectively. In addition, the clinical staff completed the Clinical Global Impression (CGI)-overall improvement during half day of observation of overall behaviour of the child while completing various tasks in the clinic.

Genetics. The affected child, parents and unaffected siblings were invited to participate in the genetic component of the study. DNA was extracted from a blood or saliva sample for each participant, if the subject was only amenable to the latter. A panel of 8 SNPs (rs2036212, rs2809823, rs2809822, rs2809819, rs2809818, rs2030048, rs2809817, rs2030049) were genotyped using Sequenom iPlex Gold Technology ${ }^{34}$, based on the markers previously examined ${ }^{16}$. Two SNPs failed the genotyping (rs2809822, rs2809819). For each of the rest of the SNPs, genotyping error was estimated by including duplicates of two reference samples on every plate. Genotypes for these samples were read with $100 \%$ accuracy on each of the plates, and each SNP was genotyped in more than $95 \%$ of the patients. 
Statistics. Family based tests of association were conducted using the FBAT statistical package (version 2.0.3 $)^{35}$. Family-based association analysis has two major advantages over population-based (case/control) association studies: it is not affected by population stratification, and it may have increased statistical power ${ }^{36}$. Further, because the non-transmitted parental alleles are the control alleles, this method controls for other possible sources of bias, such as socio-economic status. All the analyses were performed under the assumption of an additive model, with a null hypothesis of no linkage and no association.

In a first step, analysis was conducted with the total sample. Further analysis was conducted in two distinct groups: (1) Families where mother was exposed to no/mild stress during pregnancy (examples of mild stressors include minor financial worries and arguments with friends); (2) Families where mother was exposed to moderate/extreme stress during pregnancy (examples of these stressors include major financial problems, physical/sexual abuse, and imprisonment/abandonment of partner). Significance level was set at $\mathrm{P}<0.05$. Due to the exploratory nature of this study, no correction for multiple-testing was performed. When the total sample was stratified based on maternal stress during pregnancy, SNP rs2036212 was not informative due to lack of the minimum number of families $(n=10)$ required.

In a specific case of the FBAT (where both parents are known, and when the additive model is used), the $Z^{2}$ statistic can be considered equivalent to a $\chi^{2}$ TDT statistic ( $N$. Laird, personal communication). FBAT is an extension of McNemar's test used to calculate transmission disequilibrium in a pedigree, where $\chi^{2}{ }_{T D T}=$ $(\mathrm{T}-\mathrm{NT})^{2} /(\mathrm{T}+\mathrm{NT})$. $\mathrm{T}$ and NT denote the number of transmissions and non-transmissions of a specific allele from the parent to the affected offspring. In order to obtain an estimate of effect size, we applied this generalization and calculated the effect size $\Phi$ as for a $\chi^{2}$ test, where the following formula is used $\Phi$ = square root $\left[\chi^{2} / \mathrm{N}(\mathrm{k}-1)\right]$, where $\mathrm{N}=$ sample number, and $\mathrm{k}=$ number of rows or columns or 2 in the McNemar's test. The number of informative families was used to calculate N. Effect sizes of $0.1,0.3$, and 0.5 are considered small, medium and large respectively (Suppl. Table S3).

\section{Compliance with ethical standards.}

All animal procedures in this report were conducted in accordance with the guidelines of the Canadian Council of Animal Care and by the Animal Care Committees of McGill University/Douglas Mental Health University Institute. The human research protocol was approved by the DMHUI Research Ethics Board. Parents were explained the study and provided written consent. Children (affected child and unaffected siblings) were explained the study and gave their assent to participate. Children with an IQ less than 70, and/or a diagnosis of either Tourette syndrome, pervasive developmental disorder, or psychosis were excluded from the study.

\section{Results}

Gpr88 knockout mice (or Gpr88-/- mice) are impaired in the Five-Choice Serial Reaction Time Task (5CSRTT). 
The 5-CSRTT (Suppl. Fig. S1) evaluates all three features of ADHD, namely hyperactivity, inattention and impulsivity ${ }^{37}$, and is considered most translatable between humans and rodents ${ }^{38}$. In this test, Gpr88 ${ }^{-/-}$mice were able to learn the task normally (Suppl. Fig. S2). However, at testing (Fig. 2A and statistics in Suppl. Table S1), mutant mice displayed reduced accurate responding, which requires spatial and temporal attention, and this was observed across the four stimulus presentation times. Further, more omission errors were found at shorter intervals, indicating that attention deficits relate to task difficulty. $\mathrm{Gpr}^{88^{-/}}$mice also made more responses prior to signal presentation (premature responses) during the $5 \mathrm{~s}$ period preceding stimulus presentation, an index of higher motor impulsivity. Finally, Gpr88 ${ }^{-/}$mice showed increased beam breaks (activity), confirming hyperactivity in these mice. Otherwise, reward collection latency was higher or unchanged, suggesting intact motivation to retrieve the food. The absence of GPR88 in mice therefore decreases attention, increases motor impulsivity, and produces hyperactivity in the 5-CSRTT.

\section{Gpr88 knockout mice are impaired in the Attentional Set Shifting task (ASST).}

The ASST, based on human cognitive testing ${ }^{39}$, is also used in rodents ${ }^{40}$. The paradigm involves seven successive steps of increasing difficulty, where an odor and/or a medium are associated with a reward (Suppl. Fig. S3). Data for three parameters are shown in Fig. 2B and statistics are in Suppl. Table S1. In the first three steps of the procedure, mutant mice made more wrong trials in compound discrimination (CD) and reversal 1 (R1) steps, and showed higher average time to choose in the simple discrimination

(SD) task, suggesting lower capability to maintain attention. Importantly, Gpr88 ${ }^{-/-}$mice were slower in acquiring the last task (extra-dimensional shift, EDS) considered most meaningful, and this was observed for all the parameters (number of trials, wrong trials and latency to choice). Lack of GPR88 in mice therefore reduces attentional performance in the ASST.

\section{Gpr88 knockout mice are impaired in the Go/No-Go task}

This task ${ }^{41}$ was designed to detect deficient inhibitory processes, which characterize exacerbated impulsivity in children with ADHD, and was successfully transposed to rodents ${ }^{42}$ (Suppl. Fig. S4). In the conditioning phase (Suppl. Fig. S5), Gpr88 ${ }^{-/-}$mice showed a learning pattern similar to $\mathrm{Gpr} 8^{+/+}$controls, suggesting that the motivational state was intact. In the Go phase (Fig. 3A and statistics in Suppl. Table S1), the two groups similarly used fewer nosepokes to earn more rewards between first and last trials, indicating normal learning of the task. However, Gpr88 ${ }^{-/-}$mice showed significantly more premature responses, demonstrating lower ability to refrain from initiating an action. Despite higher premature responding, mutant mice eventually reached the criterion and moved to the next Go/No-Go step. In this phase success rates for the Go trials remained stable (Suppl. Fig. S6), however, Gpr88 ${ }^{-/-}$mice showed higher number of premature responses, more commission errors (inability to refrain from inappropriate responding) and a higher impulsivity index (Fig. 3B and statistics in Suppl. Table S1). Thus, the absence of GPR88 in mice increases motor impulsivity in the Go/No-Go task. 
We also treated Gpr88 ${ }^{-/-}$mice and their controls with atomoxetine (Suppl. Fig. S7), a noradrenaline reuptake blocker used clinically to treat both adult and childhood $\mathrm{ADHD}^{43}$. In the saline groups, as expected, the percent of premature responses was increased in $\mathrm{Gpr} 88^{-/-}$mice compared to Gpr88 ${ }^{-/}$controls. Atomoxetine $(10 \mathrm{mg} / \mathrm{kg})$ caused a drastic reduction in the percentage of premature responses in both genotypes, and the increased impulsivity detected in $\mathrm{Gpr}^{-{ }^{-/}}$mice was totally abolished. The drug had no effect on accuracy indicating a preserved instrumental memory performance in atomoxetine treated mice. These data confirm predictive validity of the Go/No-Go task under our experimental conditions, and supports the notion that lack of GPR88 activity produces an ADHD-like impulsive phenotype.

\section{A2A-Gpr88 and D1-Gpr88 knockout mice are impaired in waiting and stopping impulsivity, respectively}

We previously showed dissociable roles of GPR88 at the level of MSN of the direct and indirect pathways for a number of behaviors ${ }^{11}$. To investigate whether GPR88 in D2R- and D1R-MSNs plays differential roles in the regulation of impulsive behaviors, we tested A2AR-Gpr88 (D2R pathway), and D1RGpr88 mice and their corresponding controls (A2AR-Ctl and D1R-Ctl) in the Go/No-Go task. A2AR-Gpr88, and D1R-Gpr88 mice did not differ from their controls in the conditioning phase (Suppl Fig. S8), suggestive of intact motivational state in the conditional mutants. In the Go phase, all the groups used fewer nosepokes to earn more rewards between first and last trials, indicating normal learning of the task (not shown). During testing however (Fig. 4A and Supp.I Table S1), A2AR-Gpr88 mice showed significantly more premature responses over all sessions, as well as between first and last sessions, indicating lower ability to wait during the pre-trial period. This was not the case for D1R-Gpr88 mice.

Despite higher premature responding, A2AR-Gpr88 mutant mice eventually reached the criterion of accuracy and moved to the next Go/No-Go step. In this phase (Fig. 4B-D and statistics in Suppl. Table $\mathrm{S} 1)$, accuracy for the Go trials remained stable (not shown). However again, A2AR-Gpr88, but not D1RGpr88, mice showed higher number of premature responding (Fig. 4B), confirming lower inhibition control. Interestingly, the percentage of commission errors decreased normally along sessions in A2ARGpr88 mice, similar to their controls, but remained stable in D1R-Gpr88 mice. Thus, commission errors were higher than controls in several sessions, over the last 5 sessions and when comparing first and last session, indicating failure for D1R-Gpr88 mice to refrain their impulsive behavior. This was confirmed by the impulsivity index (Fig. 4D) that was higher across sessions for D1R-Gpr88 but not A2AR-Gpr88 mice.

Overall therefore, A2AR-Gpr88 mice were unable to withhold from starting the task (higher premature anticipatory responding, waiting impulsivity) while D1R-Gpr88 mice were unable to refrain from performing the task (higher commission errors, stopping impulsivity). Together these results clearly differentiate two distinct GPR88-mediated mechanisms regulating impulsivity.

\section{The human GPR88 gene is associated with ADHD in children}


Because ADHD-like behaviors were detectable in $\mathrm{Gpr} 88^{-/-}$mice across three gold standard animal models, we next moved to humans and investigated whether genetic variants in the GPR88 gene show an association with ADHD dimensions in a clinical sample of children with ADHD (clinical characteristics in Methods).

We tested a selected panel of 6 single nucleotide polymorphisms (SNPs) for association with overall DSM-IV diagnosis, quantitative measures of behavior and cognition, and response to treatment with a fixed dose of methylphenidate (MPH) using family-based association tests (see Methods). The study included 567 nuclear families having one or more child with a DSM-IV diagnosis of ADHD ${ }^{44}$. Of the total number of children included in this family-based study, $78.1 \%$ were male; $52.9 \%$ were diagnosed with the combined subtype, while $38.1 \%$ and $9 \%$ had the inattentive and hyperactive subtypes of ADHD respectively. Among comorbid disorders, $41.9 \%$ had oppositional defiant disorder, $17.3 \%$ had conduct disorder, $43 \%$ had anxiety disorder (including phobias), and $7 \%$ had a mood disorder.

In the total sample, a significant but minor association was observed with one of the 6 SNPs in the selected panel (rs2036212, $5^{\text {'gene }^{16}}$ ) with overall DSM-IV diagnosis and dimensions of behaviour, treatment response and cognition (Table 1 and statistics in Table S3).

More interestingly, however, when the total sample was stratified based on maternal stress during pregnancy, a highly significant association was observed with tag SNP rs2809817 (3'UTR $\left.{ }^{16}\right)$ in the group where mothers experienced moderate to severe stress during pregnancy. Here, a significant overtransmission of the T allele was observed with categorical DSM-IV diagnosis ( $Z=3.41, P=0.0006)$, suggesting that this allele carries risk for ADHD. Further association was observed in the quantitative FBAT analysis. The $T$ allele showed association with: (a) total number of $A D H D$ items $(Z=2.74, P=0.006)$; (b) number of inattention $(Z=2.67, P=0.008)$, hyperactivity $(Z=2.73, P=0.006)$, and impulsivity $(Z=2.70$, $P=0.007)$ items on DISC-IV; (c) Conners' $-P(Z=2.25, P=0.02)$ and Conners' $-T(Z=2.85, P=0.004)$ scores at baseline; (d) dimensional scores on the Child Behaviour Checklist (CBCL). This suggests that this allele is associated with a more severe symptom profile as assessed in the home (Conners'-Parents, CBCL, DISC), school (Conners'-T), and clinic (DSM-IV diagnosis).

In terms of cognitive function, association was observed with several dimensions, with the T allele overtransmitted to higher number of errors: (a) spatial working memory, planning and interference control, as measured by the SOPT; (b) commission errors on the CPT; (c) non-perserverative errors on the Wisconsin Card Sorting Test. It is interesting that this tag SNP shows association not only with clinical dimensions of $A D H D$, but also with Research Domain Criteria (RDoC) within the domain of cognitive function. Interestingly, significant association was also observed with response to treatment with MPH (Table), as noted in clinic.

It is also interesting to note that when family-based analysis was conducted in the group where the mothers experienced none/mild stress during pregnancy, the T allele of tag SNP rs2809817 showed significant association with: (a) lower number of inattention items on DISC-IV (under-transmission of T 
allele; $Z=-1.99, P=0.05)$; (b) lower scores on specific dimensions on $C B C L$, particularly withdrawn behaviour ( $Z=-2.84, P=0.004)$; (c) better performance on the SOPT $(Z=-2.37, P=0.02)$. These results suggest that not having the $T$ allele at this locus may be protective on some dimensions of ADHD.

\section{Discussion}

Here we report a preclinical and a clinical study, which both address the possibility that GPR88 signaling in the brain may regulate behavioral dimensions related to ADHD. In the mouse study, mutant mice lacking the Gpr88 gene showed hyperactivity, reduced attention and higher motor impulsivity throughout three testing procedures. Deletion of Gpr88 gene in either indirect or direct pathways of the mesolimbic dopamine circuitry further revealed distinct implications of these two receptor populations in waiting and stopping impulsivity. The animal data therefore demonstrate that deficient activity of the orphan GPR88 receptor causally promotes ADHD-like behaviors, and also identify part of the circuit mechanisms underlying GPR88-regulated motor impulsivity. In the human family-based association study, conducted in a cohort of children affected with ADHD, a minor association was found for the tag SNP rs2036212 with DSM-IV diagnosis, treatment response and cognition. A much stronger association was found for another tag SNP when the patient population was stratified, suggesting that the T allele of tag SNP rs2809817 becomes a risk factor for ADHD when prenatal stress is involved. Overall, animal and human studies concur to suggest that GPR88 signaling should be considered a key factor in the etiology of ADHD.

\section{GPR88 regulates impulsive behaviors}

This is the first report indicating that GPR88 activity regulates impulsive behaviors. Prior animal research by others and us showed that the constitutive deletion of GPR88 in mice alters a number of behaviors in the adult ${ }^{1-8}$. In particular, we previously found high risk-taking behavior ${ }^{5}$ and excessive alcohol drinking ${ }^{8}$ in these mutant mice, features associated to ADHD in humans ${ }^{45,46}$. These findings led us to hypothesize that deficient inhibitory controls may contribute to their complex phenotypic pattern. In the present study, we found higher impulsivity in these mice in the 5-CSRTT (increased premature responses and omissions), and this was further confirmed in the Go/No-Go paradigm (higher premature responses in Go and NoGo, higher commission errors and impulsivity index in NoGo). These results have implications for psychiatric research, as dysfunctional GPR88 signaling may increase impulsivity, a symptom known to cross-cut a number of disease conditions, including borderline personality, obsessive compulsive behaviors, addiction and ADHD, and even adverse effects of Parkinson Disease treatment ${ }^{47}$.

It will be important to know whether GPR88-mediated impulse control is due to tonic receptor activity in the adult, or to compensatory rewiring of GPR88-expressing neural circuits during development. A partial response comes from the use of conditional Gpr88 knockout mice in the Go/NoGo experiment. In the latter mice, the genetic deletion was limited to one neuron population, namely either D1R-MSNs or D2RMSNs, and occurred later in development ${ }^{48}$. We found that the two conditional lines together recapitulate the impulsivity-related deficits observed in the germ line Gpr88 knockout mice, making unlikely the 
possibility that the constitutive mutant phenotype is entirely due to early developmental compensatory mechanisms. In the future, the novel pharmacological tools under development may definitely answer this question ${ }^{10,49,50}$.

\section{Mechanisms of GPR88-regulated impulsivity}

A2AR-Gpr88 mice (D2R cells) but not D1R-Gpr88 mice (D1 cells) showed higher premature responses in both Go and NoGo phases, suggesting a specific role of GPR88 expressed in the indirect pathway in "waiting impulsivity" or the inability to withhold from initiating the task ${ }^{38,51}$. On the contrary, D1R- but not A2A-Gpr88 mice showed higher commission errors and impulsivity index in the NoGo phase, indicative of a specific implication of GPR88 expressed in the direct pathway in "stopping impulsivity" or the inability to refrain from performing the task ${ }^{38,52}$. These results first demonstrate that GPR88 expressed in MSNs of the striatum are essential in regulating motor impulsivity. Second, the data clearly show dissociable cell-specific roles for the two striatal GPR88 populations.

The direct (D1R-MSNs) and indirect (D2R-MSNs) mesolimbic pathways are known to promote or suppress behaviour, respectively ${ }^{53,54}$. A correct balance between the two activities within cortico-basal ganglia-thalamic circuits is key to movement control, action selection and goal-directed behaviors, and has implications for several neuropsychiatric disorders, including notably impulsive-compulsive behaviors in Parkinson Disease or addiction ${ }^{55}$. Because GPR88 is expressed on these two opposing pathways, one may expect that deleting the receptor gene from one or the other circuit has contrasting effects, which we already documented ${ }^{11}$, and demonstrate again here for impulsive behaviors. At present, waiting and stopping impulsivities have been associated to ventral and dorsal striatum, respectively ${ }^{\mathbf{3 8}}$. Our data suggest that, in addition to these anatomical subdivisions, the two main striatal circuits also contribute to regulate two distinct aspects of motor impulsivity. We propose that releasing a brake from the indirect (A2AR-Gpr88 mice) or direct (D1R-Gpr88 mice) circuits alters the capacity to withhold from responding (waiting) or stop a response that has been initiated (stopping), respectively. Our data are also consistent with findings that D2/3 receptor availability is associated with waiting impulsivity ${ }^{56}$.

\section{Gpr88 mice, an animal model for ADHD?}

DSMV criteria for ADHD include three main dimensions, i. e. hyperactivity, inattention and impulsivity, and several behavioral testing paradigms are able to investigate these different aspects of the disease, in both humans and rodents (Fig. 1). In animals ${ }^{42}$, hyperactivity can be detected in most experimental setups, levels of attention can be measured in the 5-CSRTT and ASST, and impulsivity is most studied using the Go/NoGo task. Here we show that Gpr88 knockout mice show deficits across all the tests and, in addition, respond to atomoxetine. This genetic line could thus be considered a mouse model for ADHDlike symptoms, with both face and predictive validity ${ }^{22}$. Only few genetic mouse lines have been proposed as models for ADHD. Mice lacking the dopamine transporter are hyperactive in new environments, a phenotype reversed by psychostimulants ${ }^{57,58}$. Mice lacking the B2 subunit of the nicotinic receptor show ADHD-like deficits in behavioral flexibility and inhibitory controls ${ }^{59}$. Coloboma 
mice with mutated SNAP-25, a synaptic protein, are also hyperactive possibly due to imbalance between dopamine and noradrenaline transmission ${ }^{60}$, and show impulsivity in a delayed reinforcement task ${ }^{61}$. Finally, transgenic mice carrying a mutant human thyroid hormone receptor gene show hyperactivity, inattention and high impulsivity, and are sensitive to treatment with methylphenidate ${ }^{62}$. Gpr88 knockout mice therefore represent a novel tool in attempts to model ADHD in basic research ${ }^{63}$, with not only face and predictive validity, but also some construct validity in their alteration of dopamine transmission ${ }^{4,8}$ and cortico-striatal circuit connectivity 7,9 .

\section{A GPR88 risk allele for ADHD}

ADHD is a high heritable disorder and genetic associations studies using candidate gene and GWAS approaches have identified a number of genes ${ }^{64}$. Among these, are genes with known roles in neurodevelopment ${ }^{65}$ such as $B D N F$ or $S E M A 6 D$ for example ${ }^{66,67}$ or genes belonging to neurotransmitter systems, including glutamate (GRM1, 5 and 7), dopamine- (DRD4, DRD5), serotonin-(5HT1B, SLC6A1 and 4) and acetylcholine-(CHRNA7) related genes. Our study proposes GPR88 as another gene linked to ADHD, with reported roles in development and neurotransmission. This is the third human study supporting a genetic association between GPR88 and psychiatric disorders, and the first for ADHD. Confirmation is needed, using larger independent replication cohorts.

Two tag SNPs, located in regulatory regions of the GPR88 gene ${ }^{16}$ were significantly associated with ADHD in multiple parameters of our evaluations. The weak association for the $C$ allele of tag SNP rs2036212 in the total human cohort, versus the strong ADHD-like phenotype observed in mice, is not unexpected as the variant (or a causal variant in linkage disequilibrium with this SNP) in humans may have less drastic consequences than the extreme Gpr88 mutation (null allele) in a highly homogenous mouse.

The association was much stronger when maternal stress during pregnancy is involved, and here we identified the tag SNP rs2809817 as a risk allele for ADHD upon prenatal stress. Substantial literature has documented the importance of environmental factors in the development and persistence of $\mathrm{ADHD}^{44,68,69}$, including maternal mental health ${ }^{70}$. The compelling gene $\mathrm{x}$ environment interaction (stress $x$ GPR88) detected in the human study, highlights a potentially key role of life trajectories to modulate GPR88 function, a factor to be considered for future diagnostic and treatment strategies of ADHD.

\section{Conclusion}

Although mouse models are highly reductionists, it is remarkable that detection of an ADHD-like phenotype in mice lacking the Gpr88 gene indeed parallels a significant association in a human cohort. In fact, our mechanistic data from multiple mutant Gpr88 mice, combined with the association of GPR88 with multiple dimensions of ADHD in our cohort, strongly support a role for the receptor gene in the etiology of ADHD. It may be of interest to back-translate the human results in the mouse model, and further determine whether a causal link exists between GPR88 activity and prenatal stress in ADHD. 


\section{Declarations}

\section{Acknowledgments}

S.B.H. and BLK designed and led the project, analyzed and interpreted the data and wrote the manuscript. S.B.H., E.C., M. M., E.M. performed mouse behavioral experiments. E.D. contributed to the project design and edited the manuscript. S.M.S., M.T.S., N.G., and R.J. conceived and designed the genetic/clinical study. S.M.S., M.E.F., M.T.S., N.G. and R.J. performed and analyzed the genetic data. S.M.S. and M.T.S. interpreted the genetic/clinical data and edited the manuscript. All authors gave final approval of the version submitted.

This work was supported by the National Institute of Health (National Institute on Alcohol Abuse and Alcoholism, Grant No. 16658), the Canada Fund for Innovation and the Canada Research Chair to BLK. The work was also supported in part by grants from the Fonds de la recherche en santé du Québec and the Canadian Institutes of Health Research to RJ and NG. SMS is a recipient of the Young Investigator Award from the Brain and Behavior Foundation (NARSAD) and Dr. Mortimer D. Sackler Developmental Psychology Investigator Awards. We thank the staff at the animal facility of the Neurophenotyping Center of the Douglas Mental Health University Institute (Montréal, Canada). We also thank present and past members of the ADHD group for technical and clinical assistance.

\section{Conflict of interests}

S.B.H., S.M.S., E.C., M.M., E.M., M.M., E.D., M.T-S., M-E. F. and B.L.K. report no biomedical financial interests or potential conflicts of interest. Dr. Natalie Grizenko reports receiving research funding from $\mathrm{CIHR}$ and is a member of the advisory board for Purdue and Shire. Dr. Ridha Joober reports having received research funding from CIHR. He is on the advisory boards and speakers' bureaus of Pfizer, Janssen Ortho, BMS, Sunovion, Otsuka, Lundbeck, Perdue and Myelin. He has received grant funding from them and from AstraZeneca and HLS. He has received honoraria from Janssen Canada, Shire, Lundbeck, Otsuka, Pfizer and from Perdue for CME presentations and royalties for Henry Stewart talks.

\section{References}

1. Ehrlich AT, Semache M, Bailly J, Wojcik S, Arefin TM, Colley C et al. Mapping GPR88-Venus illuminates a novel role for GPR88 in sensory processing. Brain Struct Funct 2018; 223(3): 12751296.

2. Massart R, Mignon V, Stanic J, Munoz-Tello P, Becker JA, Kieffer BL et al. Developmental and adult expression patterns of the G-protein-coupled receptor GPR88 in the rat: Establishment of a dual nuclear-cytoplasmic localization. J Comp Neuro/ 2016; 524(14): 2776-2802.

3. Logue SF, Grauer SM, Paulsen J, Graf R, Taylor N, Sung MA et al. The orphan GPCR, GPR88, modulates function of the striatal dopamine system: a possible therapeutic target for psychiatric disorders? Mol Cell Neurosci 2009; 42(4): 438-447. 
4. Quintana A, Sanz E, Wang W, Storey GP, Guler AD, Wanat MJ et al. Lack of GPR88 enhances medium spiny neuron activity and alters motor- and cue-dependent behaviors. Nat Neurosci 2012; 15(11): 1547-1555.

5. Meirsman AC, Le Merrer J, Pellissier LP, Diaz J, Clesse D, Kieffer BL et al. Mice Lacking GPR88 Show Motor Deficit, Improved Spatial Learning, and Low Anxiety Reversed by Delta Opioid Antagonist. Biol Psychiatry 2016; 79(11): 917-927.

6. Maroteaux G, Arefin TM, Harsan LA, Darcq E, Ben Hamida S, Kieffer BL. Lack of anticipatory behavior in Gpr88 knockout mice showed by automatized home cage phenotyping. Genes Brain Behav 2018; 17(8): e12473.

7. Thomson DM, Openshaw RL, Mitchell EJ, Kouskou M, Millan MJ, Mannoury la Cour C et al. Impaired working memory, cognitive flexibility and reward processing in mice genetically lacking Gpr88: Evidence for a key role for Gpr88 in multiple cortico-striatal-thalamic circuits. Genes Brain Behav 2021; 20(2): e12710.

8. Ben Hamida S, Mendonca-Netto S, Arefin TM, Nasseef MT, Boulos LJ, McNicholas M et al. Increased Alcohol Seeking in Mice Lacking Gpr88 Involves Dysfunctional Mesocorticolimbic Networks. Biol Psychiatry 2018; 84(3): 202-212.

9. Arefin TM, Mechling AE, Meirsman AC, Bienert T, Hubner NS, Lee HL et al. Remodeling of Sensorimotor Brain Connectivity in Gpr88-Deficient Mice. Brain Connect 2017; 7(8): 526-540.

10. Jin C, Decker AM, Makhijani VH, Besheer J, Darcq E, Kieffer BL et al. Discovery of a Potent, Selective, and Brain-Penetrant Small Molecule that Activates the Orphan Receptor GPR88 and Reduces Alcohol Intake. J Med Chem 2018; 61(15): 6748-6758.

11. Meirsman AC, Ben Hamida S, Clarke E, de Kerchove d'Exaerde A, Darcq E, Kieffer BL. GPR88 in D1RType and D2R-Type Medium Spiny Neurons Differentially Regulates Affective and Motor Behavior. eNeuro 2019; 6(4).

12. Meirsman AC, de Kerchove d'Exaerde A, Kieffer BL, Ouagazzal AM. GPR88 in A2A receptor-expressing neurons modulates locomotor response to dopamine agonists but not sensorimotor gating. Eur $J$ Neurosci 2017; 46(4): 2026-2034.

13. Meirsman AC, Robe A, de Kerchove d'Exaerde A, Kieffer BL. GPR88 in A2AR Neurons Enhances Anxiety-Like Behaviors. eNeuro 2016; 3(4).

14. Alkufri F, Shaag A, Abu-Libdeh B, Elpeleg O. Deleterious mutation in GPR88 is associated with chorea, speech delay, and learning disabilities. Neurol Genet 2016; 2(3): e64.

15. Del Zompo M, Severino G, Ardau R, Chillotti C, Piccardi M, Dib C et al. Genome-scan for bipolar disorder with sib-pair families in the Sardinian population: a new susceptibility locus on chromosome 1p22-p21? Am J Med Genet B Neuropsychiatr Genet 2010; 153B(6): 1200-1208.

16. Del Zompo M, Deleuze JF, Chillotti C, Cousin E, Niehaus D, Ebstein RP et al. Association study in three different populations between the GPR88 gene and major psychoses. Mol Genet Genomic Med 2014; 2(2): 152-159. 
17. Yang J, Liu A, He I, Bai Y. Bioinformatics Analysis Revealed Novel 3'UTR Variants Associated with Intellectual Disability. Genes (Basel) 2020; 11(9).

18. Willcutt EG. The prevalence of DSM-IV attention-deficit/hyperactivity disorder: a meta-analytic review. Neurotherapeutics 2012; 9(3): 490-499.

19. Barkley RA. Adolescents with attention-deficit/hyperactivity disorder: an overview of empirically based treatments. J Psychiatr Pract 2004; 10(1): 39-56.

20. Magnin E, Maurs C. Attention-deficit/hyperactivity disorder during adulthood. Rev Neurol (Paris) 2017; 173(7-8): 506-515.

21. Sharma A, Couture J. A review of the pathophysiology, etiology, and treatment of attention-deficit hyperactivity disorder (ADHD). Ann Pharmacother 2014; 48(2): 209-225.

22. Sontag TA, Tucha O, Walitza S, Lange KW. Animal models of attention deficit/hyperactivity disorder (ADHD): a critical review. Atten Defic Hyperact Disord 2010; 2(1): 1-20.

23. Grizenko N, Lachance M, Collard V, Lageix P, Baron C, Ben AL et al. Sensitivity of tests to assess improvement in ADHD symptomatology. Can Child Adolesc Psychiatr Rev 2004; 13(2): 36-39.

24. Shaffer D, Fisher P, Lucas CP, Dulcan MK, Schwab-Stone ME. NIMH Diagnostic Interview Schedule for Children Version IV (NIMH DISC-IV): description, differences from previous versions, and reliability of some common diagnoses. J Am Acad Child Adolesc Psychiatry 2000; 39(1): 28-38.

25. Conners CK, Sitarenios G, Parker JD, Epstein JN. Revision and restandardization of the Conners Teacher Rating Scale (CTRS-R): factor structure, reliability, and criterion validity. J Abnorm Child Psychol 1998; 26(4): 279-291.

26. Conners CK, Sitarenios G, Parker JD, Epstein JN. The revised Conners' Parent Rating Scale (CPRS-R): factor structure, reliability, and criterion validity. J Abnorm Child Psychol 1998; 26(4): 257-268.

27. Achenbach TM. Manual for the Child Behavioral Checklist/4-18 and 1991 Profile. University of Vermont Department of Psychiatry: Burlington, VT, 1991.

28. Conners CK. The computerized continuous performance test. Psychopharmacol Bull 1985; 21(4): 891-892.

29. Petrides M, Milner B. Deficits on subject-ordered tasks after frontal- and temporal-lobe lesions in man. Neuropsychologia 1982; 20(3): 249-262.

30. Shallice T. Specific impairments of planning. Philos Trans R Soc Lond B Biol Sci 1982; 298(1089): 199-209.

31. Taerk E, Grizenko N, Ben Amor L, Lageix P, Mbekou V, Deguzman R et al. Catechol-Omethyltransferase (COMT) Val108/158 Met polymorphism does not modulate executive function in children with ADHD. BMC Med Genet 2004; 5: 30.

32. Wechsler D. Manual for the Wechsler Intelligence Scale for Children. 3rd edn. The Psychological Corporation: Oxford (UK), 1991.

33. McNeil T, K S. McNeil-Sjostrom Scale for Obstetric Complications. Lund University: Lund University; 1995. 
34. Ehrich M, Bocker S, van den Boom D. Multiplexed discovery of sequence polymorphisms using basespecific cleavage and MALDI-TOF MS. Nucleic Acids Res 2005; 33(4): e38.

35. Laird NM, Horvath S, Xu X. Implementing a unified approach to family-based tests of association. Genet Epidemiol 2000; 19 Suppl 1:S36-42:: S36-S42.

36. Haldar T, Ghosh S. Power comparison between population-based case-control studies and familybased transmission-disequilibrium tests: An empirical study. Indian J Hum Genet 2011; 17 Suppl 1:S27-31.: S27-S31.

37. Robbins TW. The 5-choice serial reaction time task: behavioural pharmacology and functional neurochemistry. Psychopharmacology (Berl) 2002; 163(3-4): 362-380.

38. Dalley JW, Robbins TW. Fractionating impulsivity: neuropsychiatric implications. Nat Rev Neurosci 2017; 18(3): 158-171.

39. Owen AM, Roberts AC, Polkey CE, Sahakian BJ, Robbins TW. Extra-dimensional versus intradimensional set shifting performance following frontal lobe excisions, temporal lobe excisions or amygdalo-hippocampectomy in man. Neuropsychologia 1991; 29(10): 993-1006.

40. Heisler JM, Morales J, Donegan JJ, Jett JD, Redus L, O'Connor JC. The attentional set shifting task: a measure of cognitive flexibility in mice. $J$ Vis Exp 2015; (96).

41. Pennington BF, Ozonoff S. Executive functions and developmental psychopathology. J Child Psychol Psychiatry 1996; 37(1): 51-87.

42. Bari A, Robbins TW. Animal models of ADHD. Curr Top Behav Neurosci 2011; 7: 149-185.

43. Wilens TE. Mechanism of action of agents used in attention-deficit/hyperactivity disorder. $J$ Clin Psychiatry 2006; 67 Suppl 8: 32-38.

44. Sengupta SM, Grizenko N, Fortier ME, TerStepanian M, Joober R. Facing the methodological challenge in dissecting the genetics of ADHD: a case for deep phenotyping and heterogenity reduction. J Can Acad Child Adolesc Psychiatry 2020.

45. Pollak Y, Dekkers TJ, Shoham R, Huizenga HM. Risk-Taking Behavior in Attention Deficit/Hyperactivity Disorder (ADHD): a Review of Potential Underlying Mechanisms and of Interventions. Curr Psychiatry Rep 2019; 21(5): 33.

46. Luderer M, Ramos Quiroga JA, Faraone SV, Zhang James Y, Reif A. Alcohol use disorders and ADHD. Neurosci Biobehav Rev 2021; 128: 648-660.

47. Galet B, Ingallinesi M, Pegon J, Do Thi A, Ravassard P, Faucon Biguet N et al. G-protein coupled receptor 88 knockdown in the associative striatum reduces psychiatric symptoms in a translational male rat model of Parkinson disease. J Psychiatry Neurosci 2021; 46(1): E44-E55.

48. Schambra UB, Duncan GE, Breese GR, Fornaretto MG, Caron MG, Fremeau RT, Jr. Ontogeny of D1A and $\mathrm{D} 2$ dopamine receptor subtypes in rat brain using in situ hybridization and receptor binding. Neuroscience 1994; 62(1): 65-85.

49. Rahman MT, Decker AM, Langston TL, Mathews KM, Laudermilk L, Maitra R et al. Design, Synthesis, and Structure-Activity Relationship Studies of (4-Alkoxyphenyl)glycinamides and Bioisosteric 1,3,4- 
Oxadiazoles as GPR88 Agonists. J Med Chem 2020; 63(23): 14989-15012.

50. Rahman MT, Decker AM, Laudermilk L, Maitra R, Ma W, Ben Hamida S et al. Evaluation of Amide Bioisosteres Leading to 1,2,3-Triazole Containing Compounds as GPR88 Agonists: Design, Synthesis, and Structure-Activity Relationship Studies. J Med Chem 2021; 64(16): 12397-12413.

51. Dalley JW, Ersche KD. Neural circuitry and mechanisms of waiting impulsivity: relevance to addiction. Philos Trans R Soc Lond B Biol Sci 2019; 374(1766): 20180145.

52. Dalley JW, Everitt BJ, Robbins TW. Impulsivity, compulsivity, and top-down cognitive control. Neuron $2011 ; 69(4): 680-694$.

53. Cui G, Jun SB, Jin X, Pham MD, Vogel SS, Lovinger DM et al. Concurrent activation of striatal direct and indirect pathways during action initiation. Nature 2013; 494(7436): 238-242.

54. Nonomura S, Nishizawa K, Sakai Y, Kawaguchi Y, Kato S, Uchigashima M et al. Monitoring and Updating of Action Selection for Goal-Directed Behavior through the Striatal Direct and Indirect Pathways. Neuron 2018; 99(6): 1302-1314 e1305.

55. Macpherson T, Morita M, Hikida T. Striatal direct and indirect pathways control decision-making behavior. Front Psychol 2014; 5: 1301.

56. Barlow RL, Gorges M, Wearn A, Niessen HG, Kassubek J, Dalley JW et al. Ventral Striatal D2/3 Receptor Availability Is Associated with Impulsive Choice Behavior As Well As Limbic Corticostriatal Connectivity. Int J Neuropsychopharmacol 2018; 21(7): 705-715.

57. Gainetdinov RR, Caron MG. Genetics of childhood disorders: XXIV. ADHD, part 8: hyperdopaminergic mice as an animal model of ADHD. J Am Acad Child Adolesc Psychiatry 2001; 40(3): 380-382.

58. Giros B, Jaber M, Jones SR, Wightman RM, Caron MG. Hyperlocomotion and indifference to cocaine and amphetamine in mice lacking the dopamine transporter. Nature 1996; 379(6566): 606-612.

59. Granon S, Faure P, Changeux JP. Executive and social behaviors under nicotinic receptor regulation. Proc Natl Acad Sci U S A 2003; 100(16): 9596-9601.

60. Russell VA. Neurobiology of animal models of attention-deficit hyperactivity disorder. J Neurosci Methods 2007; 161(2): 185-198.

61. Bruno KJ, Freet CS, Twining RC, Egami K, Grigson PS, Hess EJ. Abnormal latent inhibition and impulsivity in coloboma mice, a model of ADHD. Neurobiol Dis 2007; 25(1): 206-216.

62. Siesser WB, Zhao J, Miller LR, Cheng SY, McDonald MP. Transgenic mice expressing a human mutant beta1 thyroid receptor are hyperactive, impulsive, and inattentive. Genes Brain Behav 2006; 5(3): 282297.

63. Rahi V, Kumar P. Animal models of attention-deficit hyperactivity disorder (ADHD). Int J Dev Neurosci 2021; 81(2): 107-124.

64. Hawi Z, Cummins TD, Tong J, Johnson B, Lau R, Samarrai W et al. The molecular genetic architecture of attention deficit hyperactivity disorder. Mol Psychiatry 2015; 20(3): 289-297.

65. Dark C, Homman-Ludiye J, Bryson-Richardson RJ. The role of ADHD associated genes in neurodevelopment. Dev Biol 2018; 438(2): 69-83. 
66. Hawi Z, Cummins TD, Tong J, Arcos-Burgos M, Zhao Q, Matthews $N$ et al. Rare DNA variants in the brain-derived neurotrophic factor gene increase risk for attention-deficit hyperactivity disorder: a nextgeneration sequencing study. Mol Psychiatry 2017; 22(4): 580-584.

67. Demontis D, Walters RK, Martin J, Mattheisen M, Als TD, Agerbo E et al. Discovery of the first genome-wide significant risk loci for attention deficit/hyperactivity disorder. Nat Genet 2019; 51(1): 63-75.

68. Brinksma DM, Hoekstra PJ, de Bildt A, Buitelaar JK, van den Hoofdakker BJ, Hartman CA et al. Parental rejection in early adolescence predicts a persistent ADHD symptom trajectory across adolescence. Eur Child Adolesc Psychiatry 2021.

69. Palladino VS, McNeill R, Reif A, Kittel-Schneider S. Genetic risk factors and gene-environment interactions in adult and childhood attention-deficit/hyperactivity disorder. Psychiatr Genet 2019; 29(3): 63-78.

70. Chen LM, Tollenaar MS, Hari Dass SA, Bouvette-Turcot AA, Pokhvisneva I, Gaudreau H et al. Maternal antenatal depression and child mental health: Moderation by genomic risk for attentiondeficit/hyperactivity disorder. Dev Psychopathol 2020; 32(5): 1810-1821.

\section{Table}

\section{Table 1. Association of GPR88 tag SNPs with ADHD, clinical/behavioural traits, response to treatment} and cognition. Schematic representation of family-based association tests (FBAT) analyses conducted in total sample (panel a), and in subgroups of families stratified based on whether the mother experienced severe/extreme stress during pregnancy (panel b), or the mother experienced minimal (or mild) stress during pregnancy (panel c). (a) In the total sample, minor association was observed between tag SNP rs2036212 (C allele) with DSM-IV diagnosis of ADHD ${ }^{44}$, clinical/behavioral measures and some cognitive measures (CPT ${ }^{28}$ ). (b) In the stress group, highly significant association was observed between tag SNP rs2809817 ( $T$ allele) with all the dimensions, i.e. clinical/behavioural traits, treatment response and cognitive dimensions, suggesting that this allele (or a variant in linkage disequilibrium with this SNP) may be a risk factor for ADHD. (c) In the no stress group, under-representation of the same rs2809817 $T$ allele was significantly associated with better performance in diagnostic, behavioural and cognitive $\left(\mathrm{SOPT}^{29}\right)$ measures. All methods are described in the Supplemental Methods section. $P$ value ranges are indicated for each measure and are color-coded (light orange, 0.01-0.049; light blue, 0.001-0.009; dark blue, 0.0001-0.0009). Under transmission is indicated by hatched boxes. DISC = Diagnostic Interview Schedule for Children; CBCL = Child Behavior Checklist; SOPT, Self-Ordered Pointing Task; CPT, Continuous Performance Test; SE, standard error; WCST = Wisconsin Card Sorting Test. 


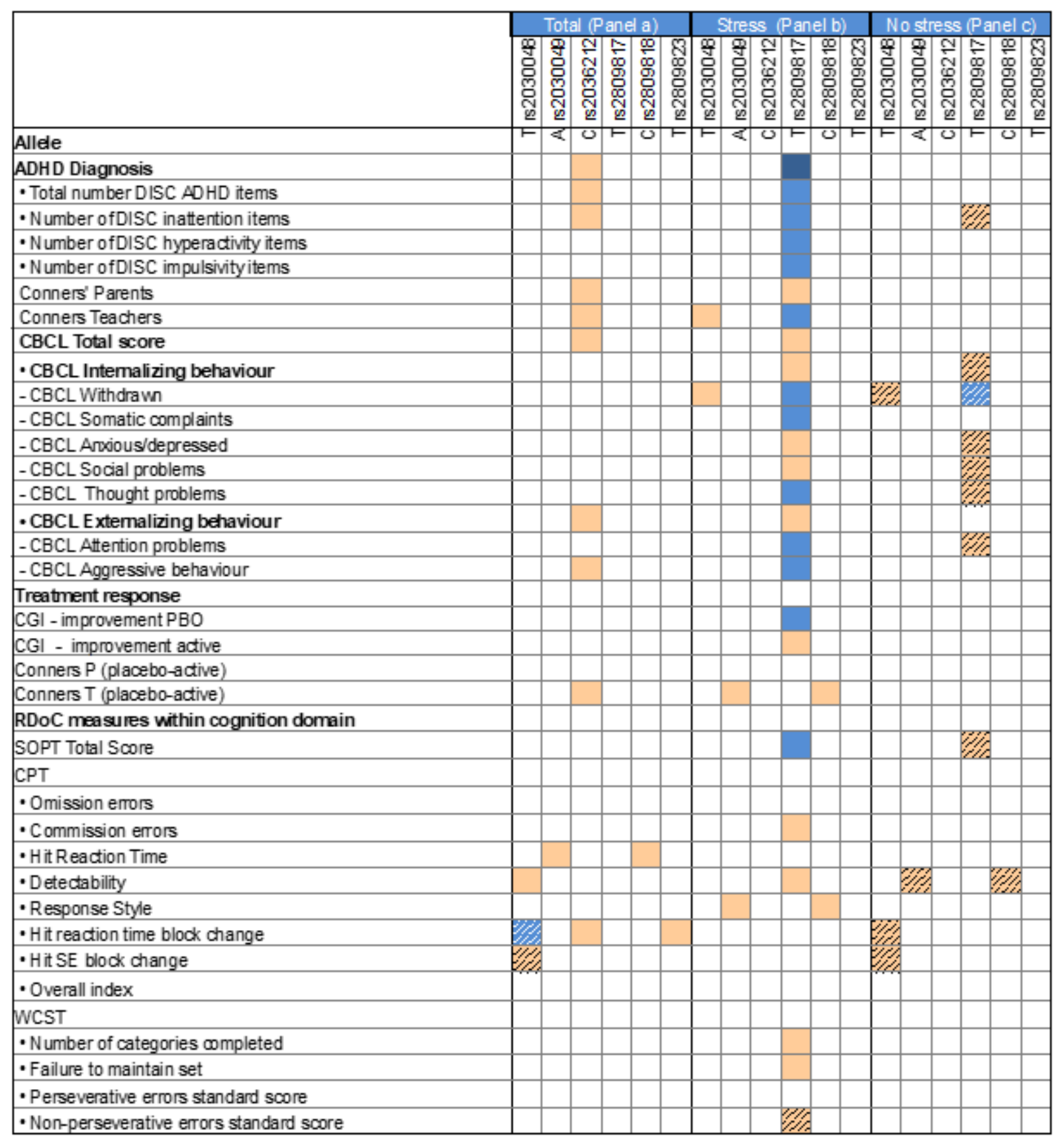

\section{Figures}




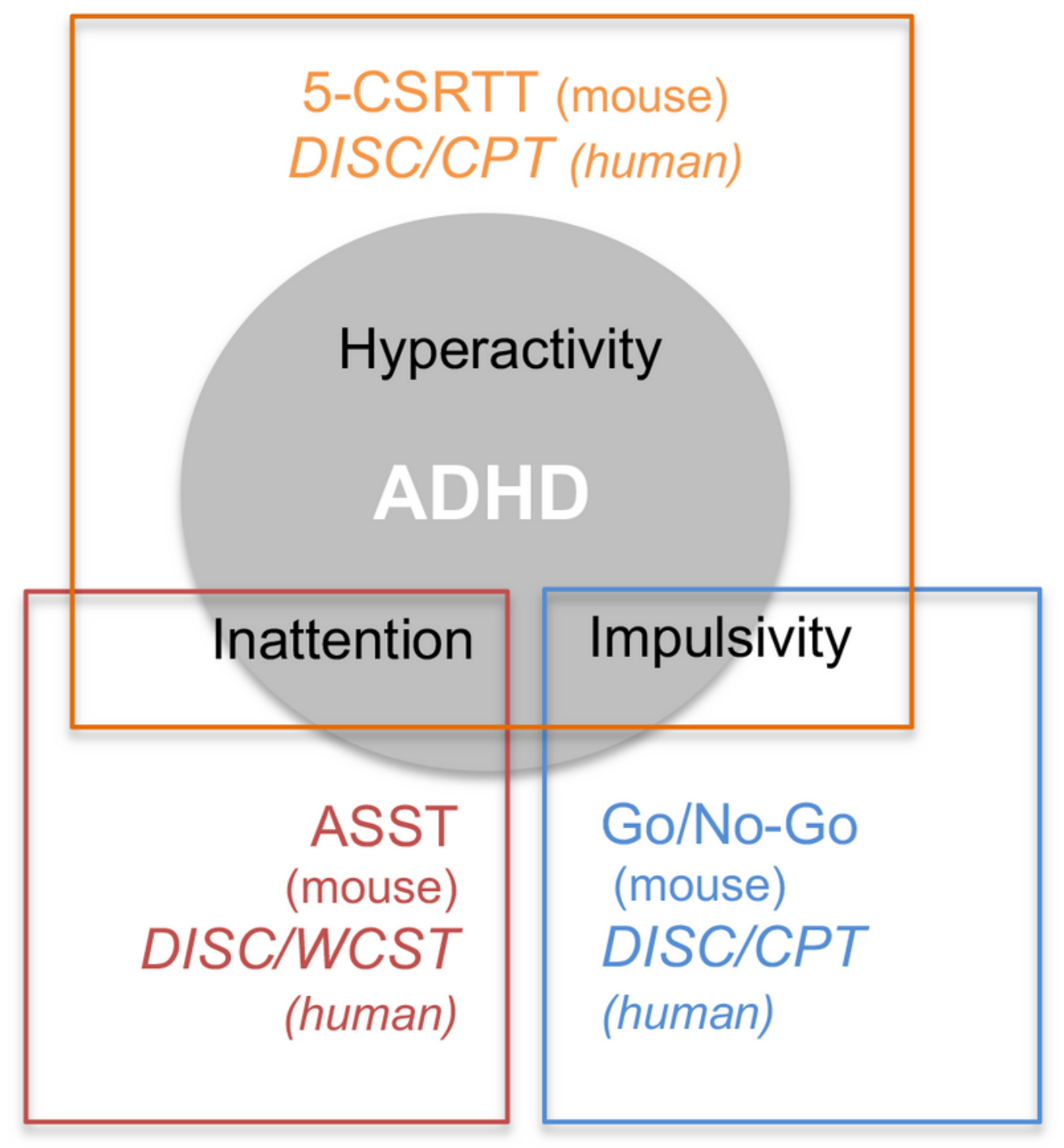

\section{Ben Hamida et al Fig. 1}

\section{Figure 1}

Cardinal features of ADHD. Three behavioral paradigms were used in this study to assess ADHD-like phenotypes in Gpr88 ${ }^{-/}$mice. The five-Choice Serial Reaction Time Task (5-CSRTT) evaluates hyperactivity, motor impulsivity and inattention, which characterize clinical features of ADHD. The Go/NoGo task and Attentional Set Shifting Task (ASST) specifically address impulsivity and inattention, respectively. Human testing paradigms, which best parallel animal testing and are used in this study 
(Table 1), are indicated in italics, and include the Diagnostic Interview for Children (DISC), the Continuous Performance Test (CPT) and the Wisconsin Card Sorting Test (WCST).
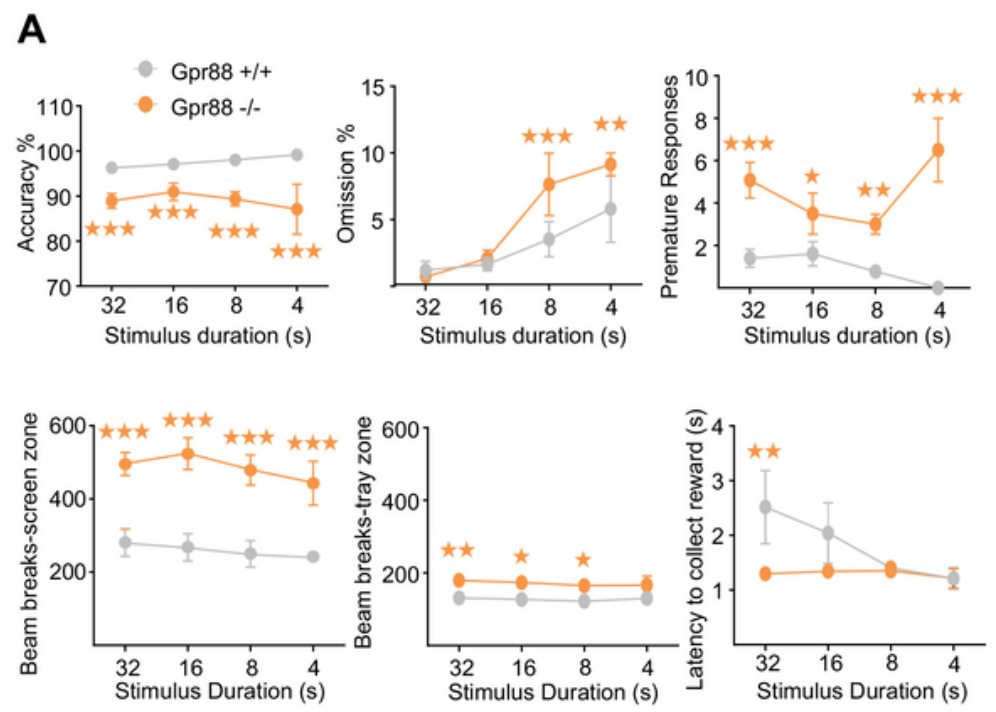

B
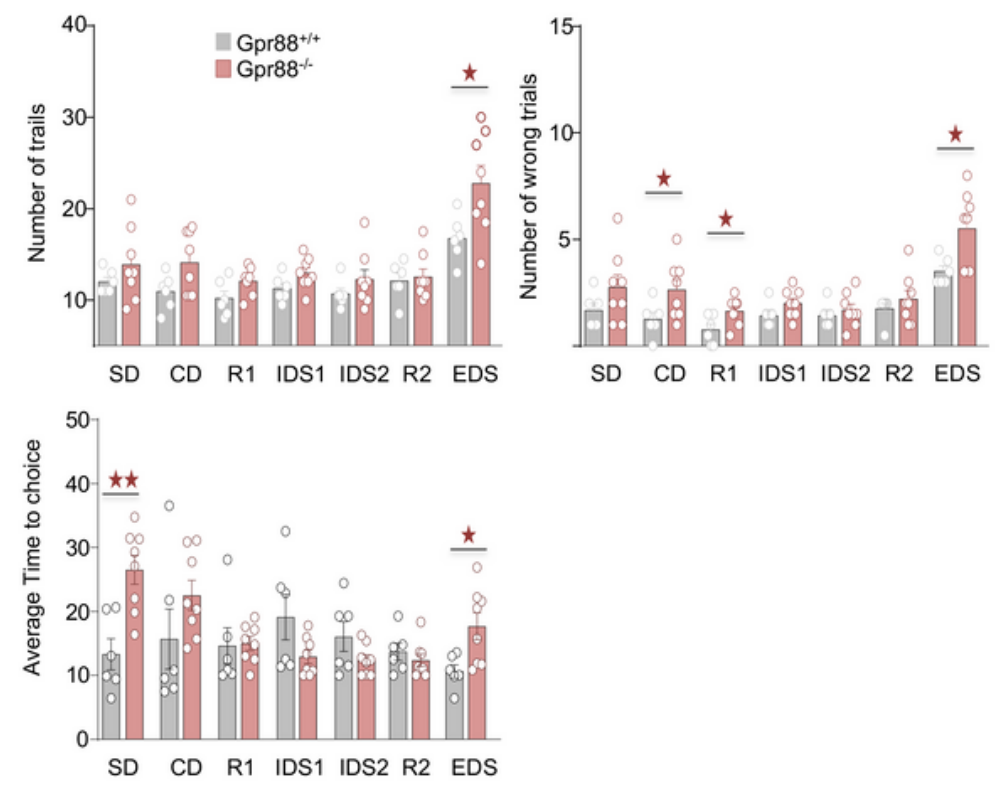

Ben Hamida et al Fig. 2

Figure 2

Gpr88 mutant mice show ADHD-like behavior in the 5-CSRTT and higher inattention in the ASST. A. Gpr88 ${ }^{-/}$mice show lower performance in all aspects of the 5-CSRTT. This test is performed in a 
touchscreen apparatus (SupplFig. S1). Mice must maintain attention to five spatial locations on a screen, and withhold from responding until a signal (flashlight) is presented randomly at one of the locations. The animal then needs to touch the screen where light was presented to obtain a food reward, a task requiring spatial and temporal attention, and effective inhibitory controls to avoid premature responding. The $\%$ accuracy was calculated as the number of correct trials divided by total number of trials $x 100$ (correct and incorrect), and the \% omission was calculated as the number of missed trials divided by number of presented trials $\times 100$. Lower accuracy and higher omissions were found across the four stimulus durations, and the number of premature responses to stimulus presentation was higher. Animal activity (infrared beams) was higher at the screen and on the reward side of the chamber. Latency to collect the reward was increased or unchanged. $n=6-8$. Grey stars show session effects; colored stars show genotype effects. ${ }^{*} p<0.05 .{ }^{* \star} p<0.01 ;{ }^{* *} p<0.001$. B. Gpr88 ${ }^{-/-}$mice show lower performance in the ASST. In this test series, mice need to associate an odor and/or a medium with a reward in seven steps of increasing difficulty (Suppl Fig. S3). Steps proceed in the following order: simple discrimination (SD), compound discrimination (CD), reversal (R1), intra-dimensional shift IDS1, IDS2, reversal (R2) and extradimensional shift (EDS). For each step, mice are required to perform eight consecutive correct responses before moving onto the next step. Gpr88 ${ }^{--}$mice needed more trials to achieve EDS, made more wrong trials in CD and EDS, and showed higher latencies to correct choice in SD and EDS. Data are represented as mean $( \pm$ SEM) per session. $n=6-8$. Stars show genotype effects * $p<0.05$. 
A
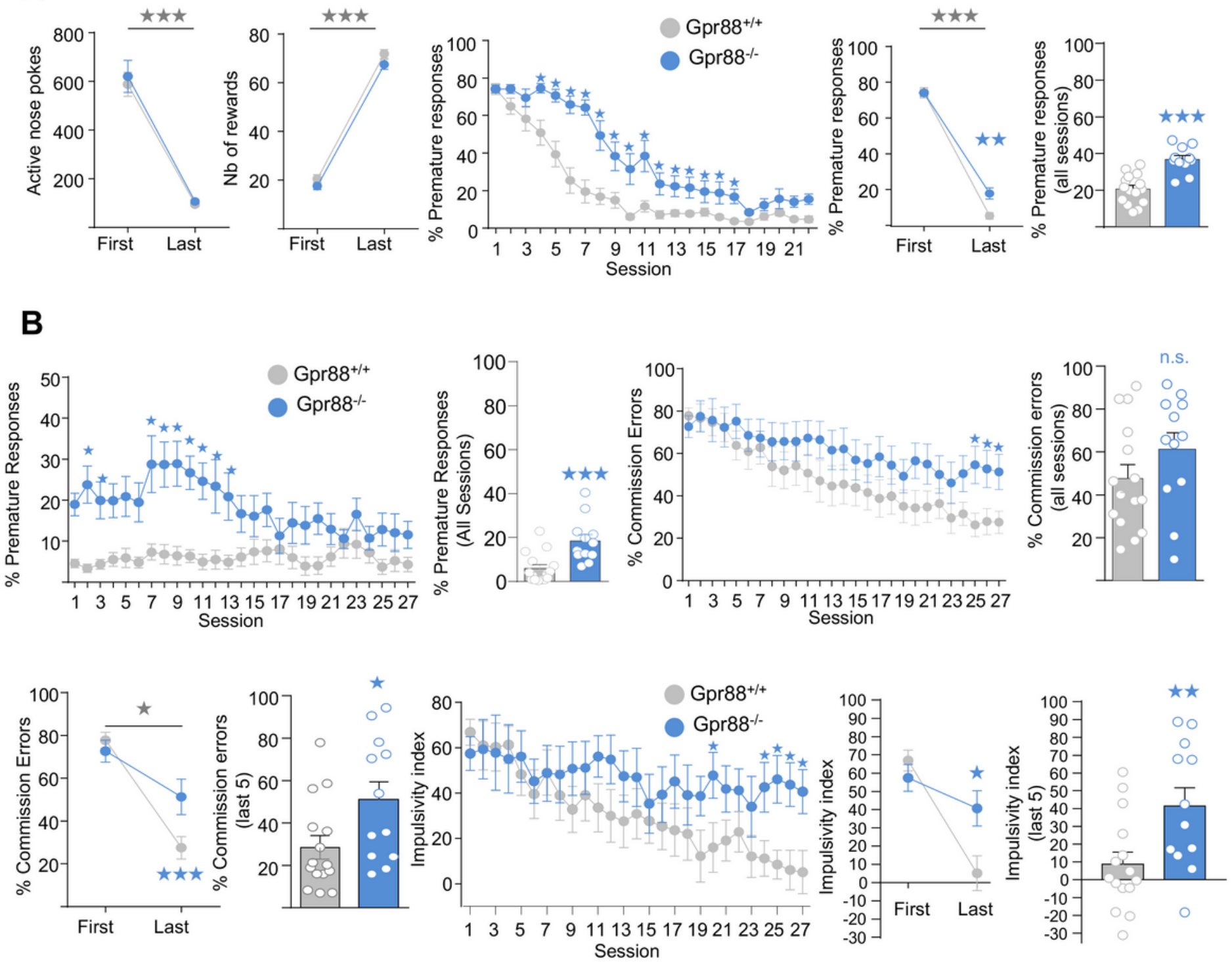

\section{Ben Hamida et al Fig. 3}

\section{Figure 3}

Gpr88 mutant mice show higher motor impulsivity in the Go/No-Go task. Mice are initially trained to nose poke for food reward, signaled by a light cue in operant boxes (autoshaping Suppl Fig. S4). Ability to inhibit the already initiated response was then measured in two successive experimental series. A. Go phase. Intertrial (no light) and pretrial (house light) periods are introduced before the cue light is illuminated, to test whether animals are able to withhold responding. Gpr88-/- mice and their controls showed similar learning patterns, shown by same numbers of active nosepokes and earned rewards during the first and last session. The \% premature responses (number of premature responses divided by number of pre-trials x 100) was increased in $\mathrm{Gpr}^{-1 /-}$ mice in sessions 4 to 11 , and between first and last 
session. B. No-Go phase. Animal's ability to inhibit inappropriate behavior was further tested. A light cue only (Go, omission error) or a paired light/sound cue (no-Go, commission error) are randomly presented and rewarded. The \% premature responses was increased for Gpr88/- mice in sessions 1, 7, 8, 9, 10 and 11 , and over all sessions. The $\%$ commission errors (number of commission errors divided by number of No-Go trials, $x 100$ ) was increased, with a significant effect in the last session compared to first session, and over the last 5 sessions. The impulsivity index (percentage of correct go trials minus percentage of correct No-Go trials) was increased, with significant difference during the last session comparing to first session and over the last 5 sessions. Data are represented as mean $( \pm S E M)$ per session. $n=12-15$. Grey stars show session effects; colored stars show genotype effects. ${ }^{*} p<0.05 .{ }^{* \star} p<0.01 ;{ }^{* \star} p<0.001$. 


\section{A}

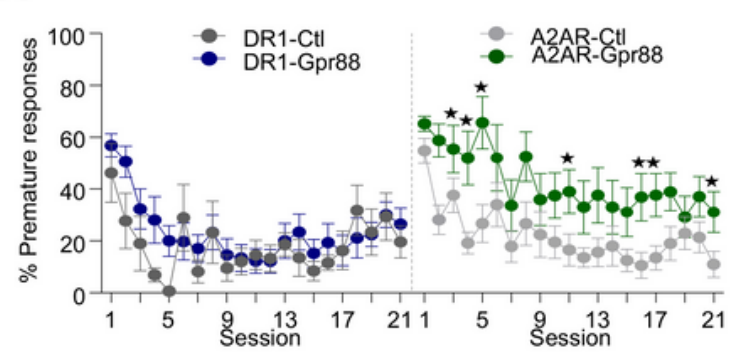

\section{B}
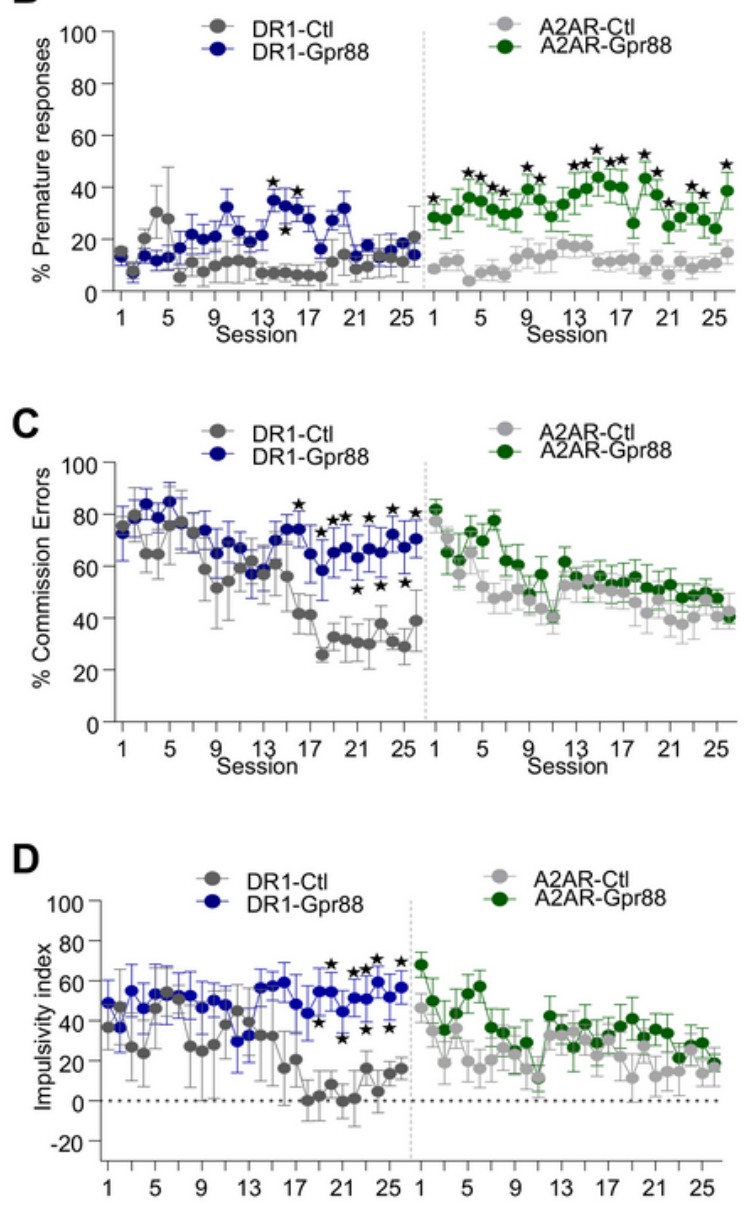

Ben Hamida et al Fig. 4
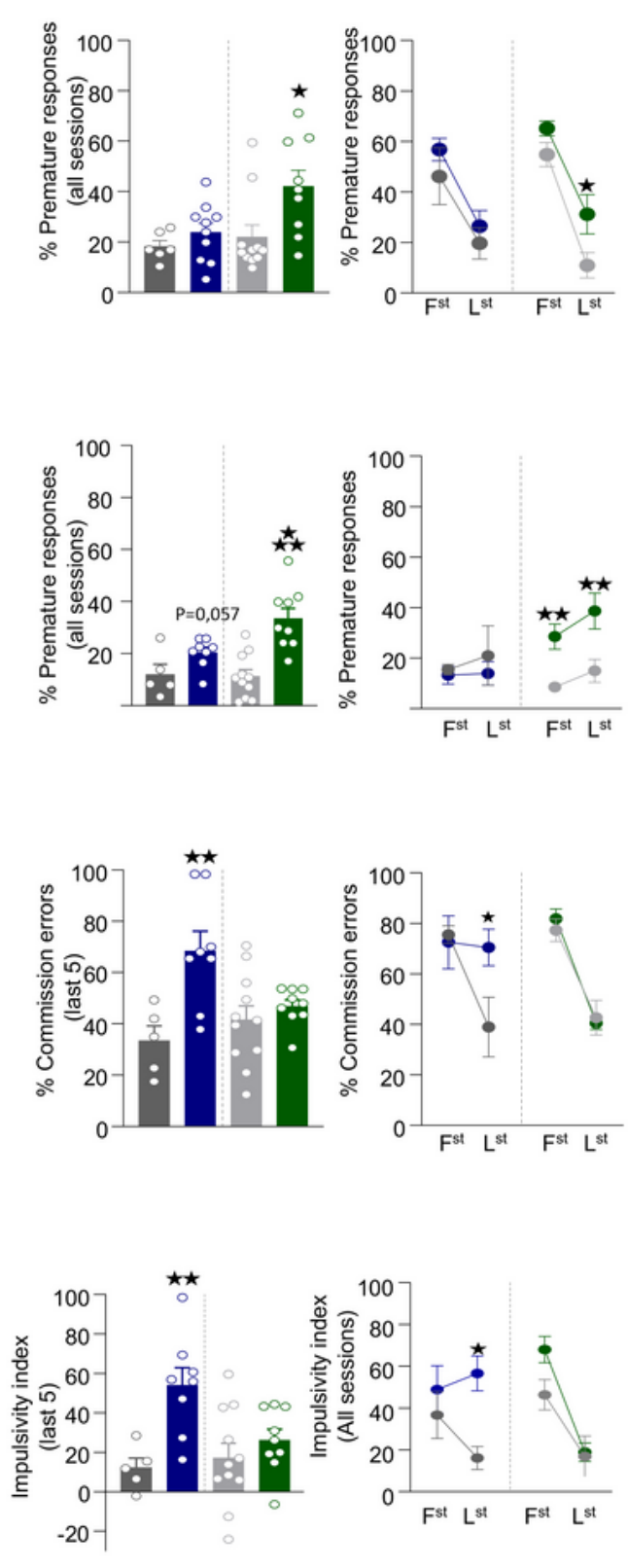

\section{Figure 4}

A2A-GPR88 and D1-GPR88 show higher waiting and stopping impulsivity, respectively, in the Go/No-Go task. The experiment was performed as in Fig. 3. A2AR- and D1R-Gpr88 mice and their respective controls (A2A-Ctl and D1R-Ctl) were initially trained to nose poke for food reward and all animals reached the criterion at the end of the AutoShaping phase (autoshaping Suppl Fig. S4). Next, mice underwent the Go phase, followed by the No-Go phase. A. Go phase. Intertrial (no light) and pretrial (house light) periods are 
introduced before the cue light is illuminated, to test whether animals are able to withhold responding. The $\%$ premature responses (number of premature responses divided by number of pre-trials $\times 100$ ) was increased in A2AR-Gpr88 but not D1R-Gpr88 mice in sessions 3-5, 11, 16-17 and 21, over all the sessions, and between first and last session. B-D No-Go phase. B. The \% premature responses was again increased A2AR-Gpr88 mice, with higher significance compared to the Go phase. D1R-Gpr88 mice showed higher premature responses in 3 session (14-16) only. C. The \% commission errors (number of commission errors divided by number of No-Go trials, $x 100$ ) and $\mathbf{D}$. The impulsivity index (percentage of correct go trials minus percentage of correct No-Go trials) were increased in DR1-Gpr88, but not D1R-Gpr88, mice over the last 5 sessions and when comparing first and last session. Data are represented as mean ( \pm SEM) per session. $n=5-11$. Grey stars show session effects; colored stars show genotype effects. * $p<0.05$. ${ }^{\star \star} p<0.01 ; * \star \star x p 0.001$.

\section{Supplementary Files}

This is a list of supplementary files associated with this preprint. Click to download.

- BenHamidaetalSupplFigures.pdf

- BenHamidaetalSuppllnformation.docx

- BenHamidaetalSupplTables.docx 